\title{
Application of Atomic Force Microscopy for Studies of Fractal and Functional Properties of Biomaterials
}

\author{
S. Kulesza ${ }^{a, *}$, M. Bramowicz ${ }^{b}$, P. CZAJA ${ }^{c}$, R. JABŁOŃSKI ${ }^{d}$, J. KropiWniCKi $^{d}$ \\ AND M. ChARKIEWICZ ${ }^{d}$ \\ ${ }^{a}$ Faculty of Mathematics and Computer Science, University of Warmia and Mazury, \\ Słoneczna 54, 10-710 Olsztyn, Poland \\ ${ }^{b}$ Faculty of Technical Sciences, University of Warmia and Mazury, \\ M. Oczapowskiego 11, 10-719 Olsztyn, Poland \\ ${ }^{c}$ Institute of Metallurgy and Materials Science, Polish Academy of Science, \\ W.S. Reymonta 25, 30-059 Kraków, Poland \\ ${ }^{d}$ ChM sp. z o.o., Lewickie 3B, 16-061 Juchnowiec Kościelny, Poland
}

\begin{abstract}
The paper presents results of numerical analysis of AFM images of a surface of sandblasted Ti6Al7Nb alloys before and after wet etching procedure usually used for preparing commercially viable dental implants. Obtained results demonstrate that etching procedure efficiently cleans the implants as it leaves almost pure $\mathrm{Ti}-\mathrm{Al}-\mathrm{Nb}$ surface with trace amounts of alkali metals and increased hydrophobicity. Apart of that, it turned out that simple statistical measures of the height variations (root mean square roughness) only slightly change upon the treatment procedure, especially for scan lengths below $20 \mu \mathrm{m}$. On the other hand, correlation analysis exhibits bifractal surface patterns composed of regular residues left on otherwise helical ridges of the base material. Etching leaves its fingerprint in fractal dimension, but not in the corner frequency.
\end{abstract}

DOI: 10.12693/APhysPolA.130.1013

PACS/topics: 06.30.Bp, 68.37.Ps, 68.35.bd, 81.05.Bx, 87.85.J-

\section{Introduction}

Application of titanium and its alloys as biomedical materials began in the 1940s in the last century [1], but it was Branemark et al. in the mid of the 1960s, who first described the phenomenon of osseointegration that triggered the works towards Ti-based implants [2]. Unceasing interest in these materials follows from their high corrosion resistance [3], beneficial mechanical properties and high wear resistance [1]. Among other titanium alloys, $\mathrm{Ti}-\mathrm{Al}-\mathrm{V}$ compounds used previously in the aviation industry were tried at first. Unfortunately, highly toxic vanadium was released in corrosive body environment, hence this element has been substituted by niobium, and obtained $\mathrm{Ti}-\mathrm{Al}-\mathrm{Nb}$ alloys proved their advantageous properties in terms of biocompatibility, wear resistance and as a possible substrate for bioactive coatings (e.g. HAP) [4].

The cover layer plays an important role in the osseointegration, because it determines the durability of a connection between an implant and surrounding tissue. From this point of view, detailed analysis of spatial characteristics of the implant's surface at various scale lengths becomes a key issue. It was previously demonstrated that these parameters correlate with particular aspects of material properties, processing history and/or performance of steel alloys [5-7], but none reported similar results for $\mathrm{Ti}-\mathrm{Al}-\mathrm{Nb}$ alloys.

\footnotetext{
*corresponding author; e-mail: kulesza@matman.uwm.edu.pl
}

This work presents results on the study of the residual surface topography of dental implants in the form of tapered, threaded post that serves as a substitute for the tooth root and are made of Ti6Al7Nb alloy (manufacturer: ChM Ltd.)

\section{Experimental}

Surfaces of the implants were subject to sandblasting with $\mathrm{Al}_{2} \mathrm{O}_{3}$ (SB) prior to wet etching in appropriate mixture of various acids to remove sandblasting residues (WE). Scanning with atomic force microscope (AFM) allowed us to reconstruct 3-dimensional surface maps, which were further processed to determine various spatial characteristics: statistical (root mean square (RMS) roughness, anisotropy ratio), fractal (fractal dimension, corner frequency) and functional (kernel roughness, peak height) at various wavelengths [8, 9]. AFM measurements were carried out using Multimode 8 instrument (Bruker) in a PeakForce QNM proprietary mode with a scan size from 5 to $50 \mu \mathrm{m}$ on the top surface of the implants. Elemental analysis was made using scanning electron microscopy/energy dispersive spectroscopy (SEM/EDS) system (EDAX) with built-in ZAF algorithm.

\section{Results}

Example AFM images of the topography and the map of adhesion forces on the surface of sand-blasted dental implants made of Ti6Al7Nb alloy before and after chemical treatment are shown in Fig. $1 \mathrm{~A}$ and $\mathrm{B}$, respectively. Presented images exhibit cone surface with marked spiral ridges placed at the lowest part of the implant. Apart 

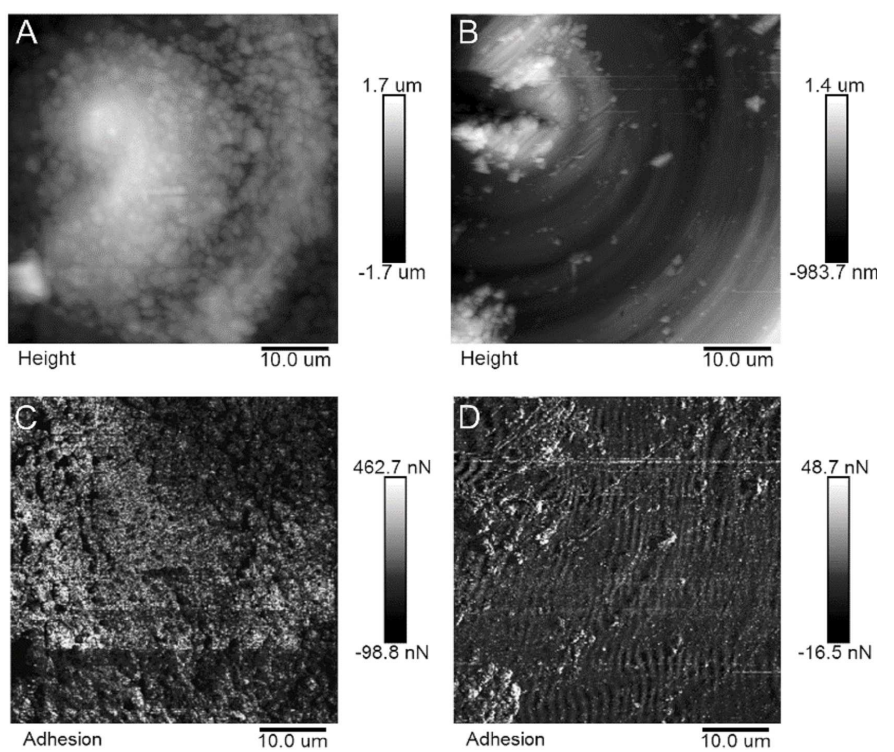

Fig. 1. AFM images of the surface topography of Ti6Al7Nb sand-blasted dental implants: (A) before and (B) after wet etching. Maps of adhesion forces between capillary layer on the surface and the scanning tip in the sand-blasted (C) and sand-blasted and wet-etched samples (D).

TABLE I

Results of elemental analysis of sand-blasted dental implants before and after chemical cleaning by means of wet etching. The numbers describe percentage contribution of a given element to the overall sample weight.

\begin{tabular}{c|c|c|c|c|c|c|c}
\hline \hline \multicolumn{3}{c|}{ sandblasting } & \multicolumn{3}{c}{ sandblasting and wet etching } \\
\hline \multicolumn{2}{c}{ base } & \multicolumn{2}{c|}{ residues } & \multicolumn{2}{c|}{ base } & \multicolumn{2}{c}{ residues } \\
\hline $\mathrm{Ti}$ & $77 \pm 2$ & $\mathrm{Ti}$ & $37 \pm 6$ & $\mathrm{Ti}$ & $86 \pm 0.2$ & $\mathrm{C}$ & $26.1 \pm 0.3$ \\
$\mathrm{Al}$ & $5 \pm 0.2$ & $\mathrm{Al}$ & $2.7 \pm 0.8$ & $\mathrm{Al}$ & $5.9 \pm 0.1$ & $\mathrm{O}$ & $37 \pm 9$ \\
$\mathrm{Nb}$ & $8 \pm 0.4$ & $\mathrm{Nb}$ & $3.0 \pm 0.4$ & $\mathrm{Nb}$ & $8.1 \pm 0.2$ & $\mathrm{Al}$ & $1.9 \pm 0.5$ \\
$\mathrm{C}$ & $10 \pm 2$ & $\mathrm{C}$ & $29 \pm 5$ & & & $\mathrm{Nb}$ & $2.6 \pm 0.7$ \\
$\mathrm{~N}$ & $4 \pm 2$ & $\mathrm{O}$ & $27 \pm 3$ & & & $\mathrm{Ti}$ & $30 \pm 9$ \\
& & $\mathrm{Si}$ & $0.6 \pm 0.1$ & & & $\mathrm{Na}$ & $1.0 \pm 0.5$ \\
& & $\mathrm{Ca}$ & $0.8 \pm 0.3$ & & & $\mathrm{Mg}$ & $0.3 \pm 0.1$ \\
& & & & & $\mathrm{Si}$ & $0.6 \pm 0.3$ \\
& & & & & $\mathrm{Cl}$ & $0.9 \pm 0.4$ \\
& & & & & $\mathrm{~K}$ & $0.5 \pm 0.2$
\end{tabular}

from flat parts of the base material, however, also tiny inclusions can be seen left behind the sandblasting process. According to elemental analysis summarized in Table I, base material after sandblasting is contaminated with small amount of carbon and nitrogen, whereas residues contain smaller amounts of $\mathrm{Al}$ and $\mathrm{Nb}$ at the expense of significant levels of $\mathrm{C}$ and $\mathrm{O}$. After wet etching, base material turns out to be free of contaminations, although remaining leftovers are still contaminated with $\mathrm{C}, \mathrm{O}$ and some trace amounts of alkali metals. Unfortunately, no clear explanation of the presence of such a large quantities of $\mathrm{C}$ and $\mathrm{O}$ contaminants can be given at this point.
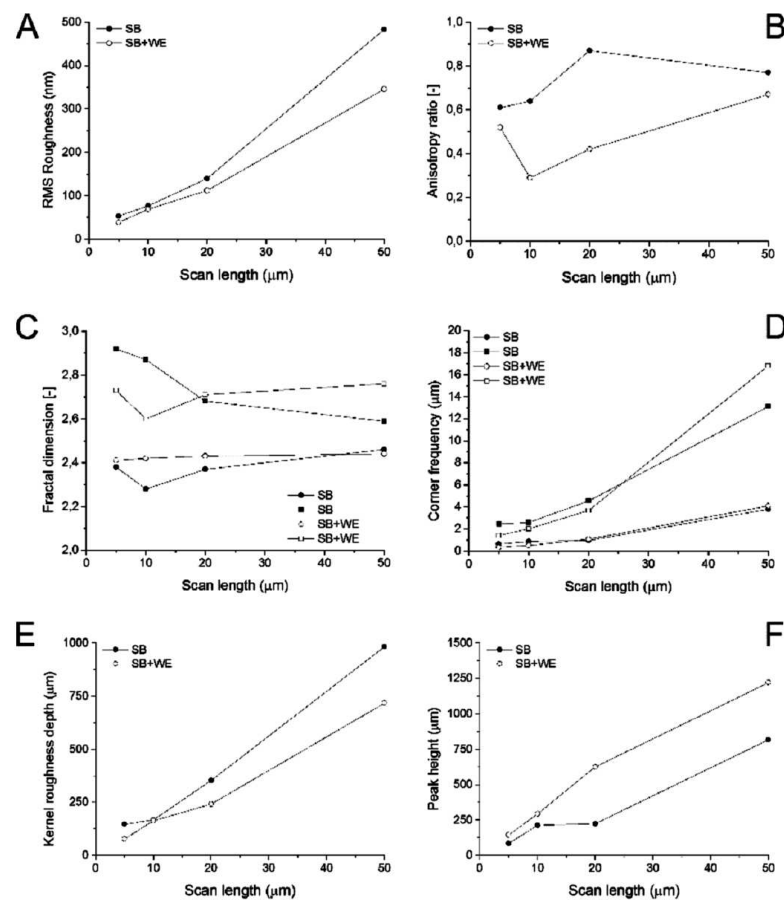

Fig. 2. Plots of spatial characteristics of surfaces of Ti6Al7Nb sand-blasted dental implants before and after chemical cleaning by means of wet etching as functions of the scan length: RMS roughness (A), surface texture ratio (B), fractal dimension (C), corner frequency (D), kernel roughness depth $(\mathrm{E})$ and peak height $(\mathrm{F})$.

Possible reason might involve the use of sticking tape in SEM imaging and analysis. On the other hand, adhesion maps shown in Fig. $1 \mathrm{C}$ and $\mathrm{D}$ prove that wet etching not only flattens the surface but also diminishes its hydrophilicity because of decreased capillary forces. Spatial characteristics of various statistical measures describing surface features are shown in Fig. 2. The plot of the RMS roughness vs. scan length (Fig. 2A) reveals power-law dependence and marked difference between sandblasted and wet-etched sampled, but only for scan lengths larger than around $15 \mathrm{\mu m}$. For smaller scan lengths, the effect of wet etching is insignificant. In turn, the plot of the surface anisotropy (Fig. 2B) exhibits huge difference introduced by the etching. For small and moderate scan lengths sandblasted sample turns out to be highly isotropic, whereas that after wet etching is found highly anisotropic. With increased scan length, however, these two characteristics approach each other in the range between 0.7 and 0.8 specific of moderately isotropic surface.

Fractal characteristics shown in Fig. 2C and D do not significantly vary with the chemical treatment. As a rule, the surface appears to be bifractal with the lower fractal dimension describing less developed surface of regular residues and the larger one that corresponds to the larger amplitude of height variations in the base material. The former becomes nearly independent of the scan length, whereas the latter clearly falls down in the same 
range. On the other hand, corner frequency is found to be governed by the power-law dependence, but remains insensitive to the chemical treatment.

Finally, functional characteristics are shown in Fig. 2E and F. As in the RMS roughness, all characteristics overlap for small scan lengths below $20 \mu \mathrm{m}$, but then become different for larger scan lengths. The plot of the kernel roughness depth (Fig. 2E) exhibits almost linear dependences on the scan length, although with the smaller slope in case of the wet-etched sample. This parameter represents the core roughness of the surface over which a load may be distributed after the surface has been flattened due to osseointegration. In turn, the plot of the peak height (Fig. 2F) shows highly non-linear dependence in the sandblasted sample, but almost linear function in the wet-etched sample. A large peak height implies a surface composed of high peaks providing high areas of contact stress when the surface is contacted, which might be beneficial for osseointegration (large amplitude of the height variations).

\section{Conclusions}

Obtained results indicate that spatial measures might vary with the scan size, so the main problem in quick characterization of the implants during the manufacturing process concerns clear definition of measurement conditions. The main finding is, however, that etching procedure is very efficient in cleaning the surface of the implant as it leaves almost pure $\mathrm{Ti}-\mathrm{Al}-\mathrm{Nb}$ surface with trace amounts of alkali metals concentrated in the very few residues.

Such statistical parameters as the RMS roughness only slightly change due to the treatment procedure and remain almost insensitive at small scan lengths (below $20 \mu \mathrm{m}$ ).
Fractal description exhibits bifractal surface arrangements of various orders composed of regular residues left on otherwise helical ridges of the base material. Etching leaves its fingerprint in fractal dimension, but not in the corner frequency.

Functional characteristics depend on the scale: below $20 \mu \mathrm{m}$ no change upon etching is observed, but above that threshold the parameters start to differ. Etching also modifies the height histogram. Increase in the core depth with increasing scan length is observed, which in general is good for loading. Similarly, large increase in the peak height is also observed, which might be beneficial for osseointegration, but not necessarily for wearing applications.

\section{References}

[1] D.B. Ratner, S.A. Hoffman, J.F. Schoen, E.J. Lemons, Biomaterials Science: An Introduction to Materials in Medicine, 3rd ed., Academic Press, San Diego 2012.

[2] P.I. Branemark, U. Breine, B. Johansson, P.J. Roylance, H. Röckert, J.M. Yoffey, Acta Anat. 59, 1 (1964).

[3] M.A. Khan, R.L. Williams, D.F. Williams, Biomaterials 17, 22 (1996).

[4] M. Niinomi, Sci. Technol. Adv. Mater. 4, 5 (2003).

[5] M. Bramowicz, S. Kulesza, G. Mrozek, Techn. Sci. 17, 4 (2014).

[6] M. Gwoździk, Z. Nitkiewicz, Archiv. Metall. Mater. 54, 1 (2009).

[7] M. Gwoździk, Z. Nitkiewicz, Opt. Appl. 39, 4 (2009).

[8] S. Kulesza, M. Bramowicz, Appl. Surf. Sci. 293, 196 (2014).

[9] M. Bramowicz, S. Kulesza, T. Lipiński, P. Szabracki, P. Piątkowski, Solid State Phenom. 203-204, 86 (2013). 